\title{
CREATIVIDAD Y VIDEOJUEGOS: NUEVOS PARADIGMAS EN LA GENERACIÓN DE APRENDIZAJE AUTÓNOMO
}

Jorge Mario Karam Rozo*

\section{Resumen}

El presente ensayo hace un análisis del trabajo desarrollado en metacognición y razonamiento espacial en juegos de computador, por el equipo de investigadores dirigido por Maldonado, $L^{1}$ y lo correlaciona con investigaciones adelantadas en otros países en cuanto a la creatividad y desarrollo del aprendizaje autónomo. Después se hace una revisión en torno a sugestivos estudios que relacionan los videojuegos con fortalecimiento del pensamiento creativo, situación que lleva a proponer que los juegos sean utilizados no sólo como herramienta sino como estrategia integral activadora de juicio de metacognición. Se destacan elementos cognoscitivos y emocionales encontrados en los videojugadores (como control de contenidos en sueños y habilidades de concentración), proponiendo el desarrollo centrado en el juego para fortalecer las estrategias activadoras de juicios de metamemoria y metacognición. Aunque se muestran varios estudios sugestivos al respecto (entre ellos uno de robótica adelantado en la Universidad Nacional de Colombia con sede en Medellín), se deja el campo abierto para la profundización y aplicación en el área específica.

Palabras clave: aprendizaje autónomo, creatividad, videojuegos, metacognición, razonamiento espacial, metamemoria.

\section{CREATIVITY AND VIDEO GAMES: NEW PARADIGMS IN GENERATING AUTONOMOUS LEARNING}

\section{Abstract}

This article examines the work developed by Maldonado et al. $\mathrm{L}^{1}$ on spatial metacognition and reasoning in computer games and correlates it with research regarding creativity and development of autonomous learning conducted in other countries. A review of suggestive studies which relate video games with the enhancement of creative thought is conducted, proposing games to be used not only as tools but also as a comprehensive strategy which fosters metacognitive judgements. Cognitive and emotional elements found in video game players (such as, controlling the contents of dreams and concentration skills) are featured and improvement of strategies focusing on games to foster metamemory and metacognitive judgements is proposed. Although several suggestive studies are shown (among them, one on robotics conducted at the Universidad Nacional de Colombia in Medellín) this field remains open to forward in depth study and application on a specific area.

Key words: autonomous learning, creativity, video games, metacognition, spatial reasoning, metamemory.

Fecha recibido: septiembre 3 de 2010 - Fecha aceptado: octubre 21 de 2010

* Psicólogo Universidad Nacional de Colombia, auditor de sistemas de garantía de calidad en salud, Universidad del Valle. Candidato a Maes- tría en Tecnologías de la información aplicadas a la educación, Universidad Pedagógica Nacional. Docente universitario y psicólogo clínico. Bogotá DC. Colombia. 


\section{Presentación}

Cierta anécdota refiere que al ser interrogado sobre qué era la inteligencia el gran Alfred Binet (creador de las famosas pruebas de IQ) sé limitó a decir: es lo que miden mis tests. Semejante respuesta que roza incluso con el cinismo y el desconcierto, sigue poniendo de relieve la complejidad del tema a abordar. ${ }^{2}$

En esencia, la definición más agradable aparece en los propios cimientos etimológicos del término atribuidos a Aristóteles, de quien se dice haber planteado que inteligencia es intelligeri, es decir leer entre líneas; una precisión astuta que revela cómo el ser inteligente tiene la posibilidad de ser trascendente con la información brindada y llevarla a límites que superen lo concreto para llegar a lo abstracto, planteamiento que J. Piaget ${ }^{3}$ llevaría a toda su extensión en el análisis epistemológico alrededor del desarrollo de la inteligencia en el sujeto.

Con H. Gardner ${ }^{4}$ las cosas tomaron un rumbo distinto, la inteligencia no era un problema monotemático, sino quepor el contrario su comprensión estaba derivada del análisis de sus múltiples modalidades, es así como gracias a este nuevo paradigma la inteligencia ahora se concibe desde distintos frentes que van desde una inteligencia cinestésica que alude a la habilidad para reproducir movimientos hasta una inteligencia emocional, tema que ha dominado el escenario psicológico en las recientes décadas.

No obstante, al lado del concepto de inteligencia se erige otro que en teoría permite que ésta pueda evolucionar, se trata del aprendizaje, elemento que en esencia permitiría que un sujeto sea capaz de superar un obstáculo que antes le impedía llegar a un determinado punto (es obvio que la consolidación del aprendizaje viene de la mano de la memoria, otro proceso psicológico no menos importantey con determinantes en su operatividad misma).

Inteligencia, aprendizaje y memoria son tres conceptos casi imposibles de desligar; es curioso que la disparidad de opiniones ha hecho que muchos autores se centren en solo algunos de ellos, obteniendo conclusiones diferentes aunque relacionadas en su fondo, en ese sentido resultan celebres las composiciones sobre el tema elaboradas por J. Piaget y L. Vigotsky ${ }^{5}$ denominadas
Lenguaje y pensamiento y Pensamiento y lenguaje respectivamente. Las propuestas computaciones al respecto tienen claros los efectos de entender estos conceptos por separado: casi se generaría un caos al intentar realizar un software que no tomara dentro de su proceso una comprensión general alrededor de los mismos; su algoritmo no estaría completo.

Sin duda el paralelismo más significativo entre procesos psíquicos y computaciones se encuentra en la aplicación de redes neuronales artificiales, un proceso que pretende establecer paralelos con el sistema nervioso biológico al intentar interactuar con los objetos del mundo real, del mismo modo que lo hace el complejo humano. ${ }^{6}$

Es precisamente en la inteligencia donde las redes neuronales solían encontrar su talón de Aquiles, si bien en tiempos recientes han surgido propuestas que permiten pensar que el paralelismo no sólo tiene lugar sino que puede llegar mucho más lejos, surge así un camino expedito para el fortalecimiento de los procesos alrededor de lo denominado como inteligencia artificial.

De hecho, resulta central intentar que un sistema no sólo pueda aprender un determinado comando (algo viable en la actualidad), sino que logre procesos metacognitivos donde refiera un aprender a aprender, proceso al que le apuestan los más modernos proyectos en ciencia cognitiva (y que a la fecha continúan reportando resultados muy significativos). ${ }^{6}$

La incorporación de la metacognición al aprendizaje surge como una inmediata necesidad a partir de la década de los ochenta, en buena medida sustentada por la sencillez que planteaba la resolución de problemas a través del modelopositivista clásico (sencillo sí, pero prácticamente incapaz de explicar procesos mucho más complejos como el aprendizaje autónomo y la toma de decisiones en la resolución de problemas).

Nada surge por generación espontánea y esto en particular es explícito en las descripciones virtuales alrededor de la inteligencia, el aprendizaje, la memoria y el lenguaje; elementos que forman un gran conjunto para describir los procesos cognitivos que intentan metaforizarse a través de complejos programas computacionales. 
La necesidad de fortalecer los programas computaciones surge de la cada vez mayor cantidad de contenidos que son vitales para comprender el mundo y adaptarse a sus diferentes apremios, como en forma clara lo señala Maldonado, Macías y otros ${ }^{1}$ en el trabajo sobre Metacognición y razonamiento espacial en juegos de computador, fuente central del presente ensayo. Investigación donde se revela cómo en la dinámica actual de los sistemas sociales se presenta un ciclo que correlaciona el cambio con la necesidad de aumentar la información disponible; ciclo que reaccionaria de manera casi viral al no encontrar límite alguno en la producción de nuevos datos para abastecer la cada vez mayor necesidad de información.

El auge del modelo computacional y la sólida interpretación en torno a un paralelismo entre el modelo neuronal y el virtual no sólo lleva a poderosos análisis en torno a conceptos tales como inteligencia artificial, sino que a su vez propende por nuevos niveles de desarrollo en torno a la búsqueda y fortalecimientos de activadores cognoscitivos capaces de ser operativos tanto en un software como en el ser humano.

De hecho la apuesta de Stenberg ${ }^{7}$ al incorporar la metacognición no sólo como una dimensión de la inteligencia sino como una herramienta de reflexión sensible de ser fortalecida, abre nuevos campos dentro del aprendizaje y por ende en la educación; pensar en programas capaces de enriquecer los elementos de autoaprendizaje y toma de decisiones, han sido el baluarte investigativo más importante durante la última década para los científicos en ciencia cognitiva (Revista Internacional de Ciencia Cognitiva, 2005). ${ }^{8}$

En ese sentido vale la pena ponderar los trabajos realizados por Maldonado y Andrade ${ }^{9}$ que encuentran en los juegos de computador una herramienta eficaz capaz de activar juicios de metacognición; propuesta que fue replicada y enriquecida con experiencias añadidas en torno a la reacción en metacognición y razonamiento espacial de estudiantes "sometidos" a emplearse con juegos de computador. ${ }^{1}$

Las experiencias de este tipo tienden a coincidir en destacar que los estudiantes con mayor estrategia en la resolución de problemas tienen más y mejores activadores de juicios de metamemoria (es decir, habilidades intrínsecas para hacer uso de su mismo proceso memorístico), planteando una correlación positiva entre desarrollo de estrategias y retención de conceptos, ${ }^{1}$ a tal punto que se arriesga la posibilidad de pensar que el fortalecimiento de estrategias incidiría en forma directa en una mejor y mayor retención de conceptos.

No obstante, ante lo anterior hay que plantear un añadido que los mismos investigadores encontraron en su estudio: al sugerirles problemas nuevos los niveles de estrategia disminuyen (un poco más en los estudiantes con menos activadores, pero a la postre disminuyen en ambos grupos); un elemento que lleva a mayor reflexión en torno a la estrategia.

¿Depende la estrategia de los activadores de metacognición y de metamemoria? Es claro que a menores niveles de juicios y activadores las estrategias disminuyen y se limitan a actos repetitivos y hasta cercanos al azar, si bien eso per se no quiere decir que se anule porcompleto la habilidad de construir estrategias para la resolución de problemas.

Lo anteriorpermite introducir una investigación reciente y bastante curiosa por demás: se trata de un trabajo realizado por la doctora Jayne Gackenbach ${ }^{10}$, psicóloga de la Universidad Gran MacEwan (Canadá) en donde manifiesta que el uso racional de videojuegos influye de manera positiva en el desarrollo de habilidades que pueden ser aplicadas en los sueños. De acuerdo con Gackenbach jugar antes de dormir parece ofrecer un inusual nivel de dominio y ajuste sobre los sueños, algo que no sólo les permitiría tener control sobre sus pesadillas sino incluso elaborar fantasías muy creativas en los mismos.

Los estudios de esta investigadora (que reconoce que fueron influenciados por un hijo ante la pasión profesada por los videojuegos) tomaron como base el hecho de que tanto los soñadores "lúcidos", sueños de los que el sujeto puede dar cuenta en forma consciente lo que en términos dinámicos se conoce como fantasías diurnas ${ }^{11}$, como en el caso de los videojugadores, se presentan mejores habilidades espaciales y facilidad de concentración.

Con el anterior paralelismo, Gackenbach encontró que las personas que juegan con frecuencia no sólo tienen 
más propensión a manifestar sueños lúcidos sino que su función creativa les permitirían en muchas ocasiones "enfrentar" sus pesadillas convirtiendo sus contenidos en tramas que con facilidad pueden ser incluso hasta divertidas.

Los estudios de esta investigadora aún se encuentran en fase inicial pero sus resultados son muy sugerentes y ya comienzan a tener eco en las comunidades científicas; incluso ya se evidencian avances terapéuticos muy significativos en el tratamiento de personas con estrés postraumático.

La experiencia con videojuegos (si bien tomada desde un contexto diferente) guarda una interesante relación con el trabajo de metacognición y razonamiento espacial en juegos de computador; en ambos casos el factor lúdico hizo parte de la estrategia de trabajo y en ambos se reportan incrementos notables de la atención (es evidente en el trabajo de Maldonado en 1999 al enfatizar en la estrategia y en los activadores).

No obstante la revisión de Maldonado y otros ${ }^{1}$ sugiere revisar las dinámicas propias de la estrategia, en tanto se evidenció que ante nuevos desafíos los niveles de estrategias adoptadas disminuían en forma considerable (sobre todo las que partían con los activadores de juicios de metamemoria), algo que enmarca un nuevo rumbo en las investigaciones sobre la influencia de los videojuegos en el aprendizaje.

Este trabajo pone especial énfasis en la elaboración individual; sin embargo, es irnportante precisar un elemento que influye en los activadores de metacognición como lo es la influencia del otro; es claro que en la búsqueda de un aprendizaje autónomo se intenta limitar al máximo la influencia conducente de un docente; pero no se puede dejar de lado la influencia que el otro puede generar de cara al aprendizaje, (elemento que desde L. Vigotsky ${ }^{5}$ se toma como un referente claro en el aprendizaje significativo y quese conceptúa como lazona de desarrollopróximoZDP).

Es curioso que en el campo de los videojuegos existió un imaginario que se convirtió en una especie de representación social; éstos fueron señalados como elementos que entorpecían la interacción social de sus usuarios, estableciendo una especie de perfil que los asumía como sujetos introvertidos y en general con pocas habilidades sociales. ${ }^{12}$

Es claro que hasta hace poco tiempo los juegos no favorecían una mayor interacción social (en especial los de consolas ya que en PC se dispone de juegos sociales casi a la par con Internet); pero esto no se correlaciona con inhabilidades sociales en sus usuarios. Al respecto Joaquín Pérez ${ }^{13}$ realiza una ponderación muy interesante al señalar el trabajo en equipo, la capacidad de superación y la destreza visual como habilidades sociales presentadas en sujetos usuarios de videojuegos (si bien también realiza un reporte no desestimable en torno a la aparición de elementos violentos no aptos para menores de edad).

Lo anterior ofrece un añadido a considerar en los estudios sobre estrategias de cara al aprendizaje autónomo: de una parte la habilidad social (que no puede ir apartada de este aprendizaje pues es claro que no siempre es sinónimo de aprendizaje independiente) plantea un proceso deteminanteen los activadores dejuicios de metamemoria y metacognición como estrategia de aprendizaje, de modo que la influencia decidida del otro se debe seguir considerando, pero no como un elemento que entorpece el aprendizaje autónomo sino como un "motivador", esto podría aumentar el rango de estrategia que se ve desfavorecido con la novedad en ejercicios. El otro aspecto (y más central ante los propósitos de investigación del autor) tiene que ver con la revisión lúdica en torno a los juegos que se utilicen de cara a la consolidación del aprendizaje autónomo; esto es asumir el juego mismocomo un activador de metamemoria y metacognición.

La diversidad existente en el ámbito de los videojuegos permite encontrar variantes de todo tipo en relación con su influencia en el surgimiento y/o consolidación de procesos creativos; es así como Shyam Sundar y Elizabeth Hutton ${ }^{14}$ encontraron una llamativa correlación entre un mejor nivel de creatividad en personas con mejor humor y mayor excitación, variables dependientes que se estimularon a través de la implementación de un conocido emulador de baile.

En la misma línea, aunque en un espacio de tipo político se expresó Toine Mander ${ }^{15}$ diputado liberal holandés que 
presentó un estudio en el cual señalaba con palabras propias como los videojuegos contribuyen al desarrollo de algunas facultades: estimulan el aprendizaje, la creatividad, la cooperación y el sentido de la innovación.

Por lo general las críticas ante los videojuegos se inclinan en señalar sus contenidos "inconvenientes" para los niños. Llama la atención que los estudios más recientes señalan la edad promedio de los usuarios por encima de los 33 años, si bien por supuesto los juegos infantiles no han desaparecido; aunque en éstos los sistemas de clasificación dejan en claro que su contenido no resulte violento de cara a los menores. ${ }^{16}$

La creatividad es un tema central dentro de los desarrolladores de videojuegos, la preocupación por elaborar contenidos novedosos y atractivos para los usuarios es una constante en sus encuentros y congresos, en todos ellos se concluye que el fomento de elementos creativos es lo más decisivo para atraer nuevos mercados y no perder a la creciente comunidad jugadora existente en la actualidad. De modo tal que la creatividad es buscada desde dos frentes: el del aprendizaje y el lúdico, que hasta hace poco se veían de manera separada, pero que en la actualidad ofrecen un modelo sugestivo de cara a fortalecer los procesos de elaboración de estrategias en el aprendizaje autónomo.

Lo anterior necesariamente propone nuevos modelos de aprendizaje que se salgan de la educación tradicional convencional (que por su misma concepción no logra avalar el aprendizaje autónomo); incorporar los videojuegos como estrategia y no sólo como instrumento, permite visualizar nuevos campos de aprendizaje.

Un atractivo trabajopropuesto en la Universidad Nacional de Medellín ${ }^{17}$ pone en tela de juicio la educación contemporánea y la distancia que en ocasiones se da en torno a los avances tecnológicos (sorprende que a la fecha algunos docentes sean tan reticentes con el uso de Intemet por parte de sus alumnos), pero lejos de quedarse en la problemática presentó un modelo de aprendizaje que se viene implementado con estudiantes de grados 10 y 11, donde aplicando conceptos y programas en robótica vieron resultados significativos tanto en las estrategias de aprendizaje autónomo como en el proceso creativo. La investigación es bastante sólida y muy diciente; sin embargo, los autores son explícitos en señalar que esto más que un nuevo paradigma se propone como complemento educativo.

Los juegos de computador (o los de consolas, depende del interés y las posibilidades de aplicación) así como las aplicaciones en robótica e inteligencia artificial deben ser vistas como algo más que un instrumento (sin que por el hecho de ser instrumentos se les restase valor alguno). Las investigaciones aquí señaladas son muy dicientes en torno a las posibilidades que éstos tienen como activadores de juicio de metamemoria y metacognición.

Un videojuego impulsa la creatividad, puede que en un momento dado la novedad implícita en los mismos mitigue la búsqueda de estrategias previas para su resolución, pero éstas no se abandonan del todo sino que por lo general luego de algunos procesos de ensayo y error terminan siendo abandonadas por otras. ${ }^{1}$

La ausencia total de estrategia es algo que con dificultad puede contemplarse en la resolución de problemas; es muy posible que los activadores de juicios de metacognición y metamemoria sean los que se alteren, aunque esto iría de la mano con el fortalecimiento del aprendizaje autónomo (y es posible que en la toma de decisiones), pero aún permanece en el terreno especulativo.

Con algunas excepciones (de obras que pertenecen a investigaciones de tipo académico) la mayoría de videojuegos que se utilizan para explorar elementos cognitivos pertenecen al terreno del entretenimiento digital, esto quiere decir que fueron diseñados en forma especial y exclusiva con el propósito de divertir; si de ahí surge un proceso de aprendizaje autónomo o consolidación de procesos creativos, se valora como un anexo y no como causa fundamental en su diseño.

Por fortuna en los años recientes el desarrollo de videojuegos viene a la par con el fortalecimiento de procesos cognitivos; es así como los emuladores han ganado fuerza y representación (en especial los que implementan esfuerzos físicos tales como emuladores deportivos o de condicionamiento corporal). 
Ya en el terreno puro cognitivo se encuentran interesantes exponentes tales como Big Brain, un divertido juego que según sus creadores mejora la inteligencia; en realidad con este juego se fortalecen ciertas habilidades (en términos de ejecución y memorización), pero de acuerdo con Adrian Owen y Grahn ${ }^{18}$ sólo las que se relacionan de manera específica con las entrenadas en el juego; en otras palabras no presenta mejora generalizada en la inteligencia. Para tal efectose llevó a cabo una gigantesca investigación con 11.430 voluntarios encontrando resultados muy dicientes en esta área.

El terreno de discusión sigue abierto, cada vez se desarrollan plataformas con propósitos educativos (el auge de los ambientes educativos b-learning y de los $e$ learning sin duda se relacionan con esto) y es importante continuar desarrollando juegos y plataformas lúdicas que permitan apreciar de manera más directa la influencia de las mismas como activadores de juicios de metamemoria y metacognición.

En ese sentido el investigador viene adelantando la gestión de una plataforma lúdica computacional como parte de su tesis de maestría, la propuesta se encamina precisamente a verificar (o refutar) los alcances que tenga un videojuego en la consolidación del aprendizaje autónomo y de la toma de decisiones; no obstante esto será tema de otro ensayo (y de la respectiva tesis de maestría, por supuesto).

De momento, se deja constancia de los significativos precedentes en el área; un campo con muchas posibilidades de exploración que sin duda presenciará avances significativos en los próximos años; docentes y estudiantes serán testigos excepcionales de la revolución educativa que se está gestando.

\section{Referencias}

1. Maldonado L, et al. (1999); Metacognición y Razonamiento Espacial en juegos de computador. Incidencia de juicios de metamemoria y sugerencias de estrategias en el aprendizaje autónomo. Bogotá: Universidad Pedagógica Nacional; 1999.
2. Gallo AJ. Medir, normalizar y excluir: los test de inteligencia. Revista Poises. 2009 Dic.; 18.

3. Piaget J. La psicología de la inteligencia. México : EdicionesCrítica; 1947.

4. Gardner H (1994) Estructuras de la mente. La teoría de las inteligencias múltiples. México : Fondo de Cultura económica; 1994.

5. Comentarios a las teorias de Jean Piaget. En: Vigotsky L. Pensamiento y lenguaje. Buenos Aires: Editorial Lautaro;1964.

6. Hilera J. Redes neuronales artificiales. Fundamentos, modelos y aplicaciones. [s.l.] : Editorial Ra-Ma, 1995.

7. Stenberg RJ. Toward triarchic theory of human Intelligence. Behav Brain Sci. $1984 ; 7(2): 269-87$.

8. Cabero Almenara J, LlorenteCejudo MC. Las TICS y la educación ambiental. Rev. latinoam. tecnol. educ. 2005; 4(2): 9-26.

9. Maldonado L, Andrade L. Ambiente computarizado para el aprendizaje autodirigido del diseño ACA2. Bogotá: Universidad Pedagógica Nacional. Proyecto de Investigación convenio Colciencias UPN; 1996.

10. Gackenbach JI. The science of lucid dreaming : the electronic media effect LDE [serie en Internet]. 2009 Jun. [citado 2011 Feb. 7 ]; 51 : [aprox. 2 p.]. Disponible en: http://www.dreaminglucid.com/issues/LDE51.pdf.

11. Freud S. (1900) La interpretación de los sueños. En: Strachey J, tr. Obras completas. Buenos Aires : Amorrortu; 1900.

12. Karam J. Erotismo y videojuegos: una nueva mirada. Rev. Perfiles. 2010 Jun.; 6 .

13. Pérez MJ. Influencia del videojuegoen la conducta y habilidades que desarrollan los videojugadores. Edutec. Revista Electrónica de Tecnologia Educativa. 2006 Jul.; 21.

14. Sundar S, Hutton E. Can video games enhance creativity?. Effects of emotion generated by Dance Dance Revolution. Creativity Research Journal. 2008; 22(3): 294-303.

15. Toine Manders T. Seguridad de los videojuegos: menos legislación y más información [monografia en Internet]. Estraburgo, Francia : Parlamento Europeo; 2009. [citado 2011 Feb 9]. Disponible en: http://www.europarl.europa.eu/ sides/get Doc.do? pub Ref=-//EP//TEXT+IM-PRESS+20090223ST $\mathrm{O} 50154+0+\mathrm{DOC}+\mathrm{XML}+\mathrm{V} 0 / / \mathrm{ES}$

16. Karam JM. (2006) La clasificación en los videojuegos: una historia de censuras, prohibiciones y propagandas [monografia en Internet]. Madrid, España : Mundogamers; 2006. [citado 2011 Feb 9]. Disponible en: http :// www.mundogamers.com/principal/articulo/52-6/la-clasificacion-en-losvideojuegos-una-historia-de-censuras-prohibiciones-y-propagandas.html

17. González J, Jiménez J, Ramírez J. Nuevos modelos de aprendizaje y desarrollo de la creatividad usando agentes robóticos. Medellín : Universidad Nacional de Colombia; 2009.

18. Owen AM, Hampshire A, Grahn JA, et al. Putting brain training to the test. Nature. 2010 Jun 10; 465(7299):775-8. 